\title{
Neurologic injury from cardiac surgery-An important but enormously complex phenomenon
}

\author{
Eugene H. Blackstone, MD
}

\footnotetext{
From the Department of Thoracic and Cardiovascular Surgery, The Cleveland Clinic Foundation, Cleveland, Ohio.

Received for publication July 18, 2000; accepted for publication July 19, 2000.

Address for reprints: Eugene $\mathrm{H}$. Blackstone, MD, The Cleveland Clinic Foundation, 9500 Euclid Ave, Desk F25, Cleveland, OH 44195 (E-mail: blackse@ ccf.org).

J Thorac Cardiovasc Surg 2003;125:S28-30 Originally published in $\mathrm{J}$ Thorac Cardiovasc Surg 2000;120:629-31.

Copyright (C) 2003 by The American Association for Thoracic Surgery

0022-5223/2003\$30.00+0

doi: $10.1067 / \mathrm{mtc} .2003 .216$
}

\begin{abstract}
Simplism
Simplism (noun): The tendency to oversimplify an issue or a problem by ignoring complexities or complications. ${ }^{1}$

In this issue of the Journal, van Dijk and his colleagues ${ }^{2}$ ask a simple question: What proportion of patients have persistent cognitive dysfunction after coronary artery bypass grafting with cardiopulmonary bypass? Their pursuit of a singlenumber simple answer began as a meta-analysis of the literature. However, they abandoned formal meta-analysis when they deemed that compatibility of studies was insufficient. Instead, they found an answer as a simple, weighted average from a handful of systematically reviewed papers.
\end{abstract}

Simple question, simple answer? Or simplistic question and simplistic answer?

\section{De Ja Vu}

As mortality and morbidity associated with cardiac injury and dysfunction have retreated in recent years, acute and persistent central nervous system injury and dysfunction after cardiac surgery have emerged as a dominating issue. However, the questions being asked about neurologic events from cardiac surgery parallel those of a generation ago, when possible injury to the heart was center stage. What is the definition of irreversible injury? Can the injury be quantified? How can irreversible or irreparable injury be distinguished from reversible phenomena? Are there bloodborne, accessible biologic markers of the injury that are reliable, specific, sensitive, and quantitative? What are the etiologies of the injury and can they be either avoided or neutralized? Are functional changes reflective of morphologic injury? What aspects of dysfunction are related to true injury and what fraction to a myriad of other aggravating factors that may be neutralized?

Neurologic injury and cognitive and behavioral dysfunction after cardiac surgery are at least as complex phenomena as myocardial injury and functional stunning. The centrality of the brain in all that it means to be human adds further complexity and confounding to the phenomena. The lesson we have learned from the phenomenon of cardiac injury is that it is possible to gain both nonspecific and mechanistic insight into the phenomenon and, thereby, at least partially avoid injury and neutralize dysfunction.

\section{Are Simple Analyses Adequate?}

Reduction of cardiac injury and management of cardiac dysfunction did not come about as the result of simple, dichotomous questions such as, "Has the heart been injured?" "Is there any cardiac dysfunction?" Instead, insight was gained when we asked, "How much?" "What is the time course?" "What are the mechanisms?" "How do quantitative markers relate to quantitative amounts of injury?"

Thus, for me, the most insightful aspect of the article by van Dijk and colleagues is their discussion of the current state of the analytic approach to neurocognitive dysfunction associated with cardiac surgery (which I would classify as the functional analogy of cardiac output, compared with structural injury per se). They point out that psychometric tests are not standardized, which poses problems of comparability. At the same time, suggesting a specific suite of standardized testing may place a straightjacket on neurocognitive research that could inhibit development of methodology to yield better insight into the nature of the phenomenon. They point 
out that the tests performed generate large quantities of data, but in the end, these data are often condensed into a simple (simplistic), information-losing format, such as a dichotomous change of one standard deviation. The authors go on to make a wonderful case for using more of the information, which is well within the capabilities of modern statistics. Yet, astonishingly, in the end, they settle for a simplistic dichotomous answer to their question, the answer to which all of us know must be, "It depends."

If we ignore the answer but pay attention to their insight, we should be stimulated to perform more appropriate analyses, as well as to seek new and better methods to quantify the injury, whether it be by neurologic testing, magnetic resonance imaging, or better serum markers than $\mathrm{S} 100 \beta$.

\section{What Is the Role of Cardiopulmonary Bypass?}

The authors are also correct in wanting to look at the contribution of such variables as support mechanisms in neurologic injury and dysfunction. Certainly, one of the factors that needs to be understood better is the contribution of cardiopulmonary bypass. The authors suggest a comparative study of on- and off-bypass coronary artery bypass grafting. This suggestion fails to take into account the complexity of that comparison. Off-bypass coronary artery bypass grafting does not simply remove a single variable (cardiopulmonary bypass) from the mix. To the contrary, off-pump coronary artery bypass grafting introduces other factors that may be associated with neurologic injury and dysfunction, including the extensive manipulation required to the heart and the use of aortic side-biting clamps.

\section{Two Methodologic Comments}

Two data-analytic issues are raised in the discussion that are important for me to reinforce. The first is the necessity to account for individual pre-test values in making group comparisons rather than simply using group means. Failure to take into account individual patient variation (preoperative test values) when making group comparisons is not simply wasteful of information, but it can lead to inaccurate inferences. However, so-called change scores are only one particular form of taking preoperative variation into account and may not be an optimal choice. This is a sophisticated statistical issue that I believe has been incompletely addressed at this time.

The second analytic issue addressed by the authors is test-retest learning. It is crucial to account for this factor, but quantifying the degree of correction necessary is challenging. The systematic direction of this bias works in such a way as to mask important effects.

\section{Meta-analysis}

Finally, the authors state they have performed a systematic literature review, not a meta-analysis. Why is the study not a meta-analysis? A "systematic review" is an overview of independent publications that uses explicit, reproducible methods and criteria to avoid bias. ${ }^{3}$ Systematic reviews can include as one of their components meta-analysis, which is a suite of mathematical and statistical procedures that integrate several independent studies that are deemed comparable.

The particular class of meta-analysis appropriate for systematic reviews is the one for which the unit of observation is the independent study. Simple pooling of information from multiple sources to obtain a so-called weighted mean, as the authors present, has been around since the 18th century, but the term meta-analysis, coined by Glass 4 in the mid-1970s, connotes a greater expectation. Meta-analysis involves meticulous, disciplined, systematic review of the literature using clear criteria for and assessment of study quality, determination of comparability among studies, and extraction of similarly defined data elements, as is exemplified by the authors. In addition to this, many of the following elements are found frequently in the subsequent quantitative integration of independent publications, that is, the meta-analysis of the information. ${ }^{5}$

- Quantitative evaluation of the diversity (heterogeneity, consistency) of results among the different studies

- Use of formal quantitative methods to explore the nature of the heterogeneity of results

- Estimation of the overall effect (fixed effect) or how it varies across studies (random effect), often employing metaregression to identify influential variables such as date of the study, differences in demography among studies, differences in other characteristics of the study population, size of the study, and in this study, average time of the post-test

- Sensitivity analyses to determine if the estimates of an effect are stable (robust)

- Formal evaluation of bias, including publication bias and study quality bias

In addition, most meta-analysis efforts would have included as wide as possible sample of studies, generally more than the final handful used in this study, accounting as best as possible for clear aspects of heterogeneity to avoid study selection bias. None of these anticipated elements of metaanalysis are contained in this paper, so I would agree with the authors that the character of the paper is one of systematic review without an accompanying meta-analysis component.

\section{Asking the Right Questions}

Thus, in the end, asking how often neurocognitive dysfunction persists after coronary artery bypass grafting with cardiopulmonary bypass, and expecting a simple one-number answer to suffice, is simplistic, however desirable. Rather, let this article be a stimulus of the development of better quantitative measures of neurologic injury and cognitive and behavior dysfunction, to the better understanding of the 
relation of neurologic injury and neuropsychiatric function, to the application of better data-analytic methodology that more fully use the precious information that is gathered, and to the development of methods to reduce injury and neutralize dysfunction, even in the absence of its complete understanding.

\section{References}

1. American Heritage Dictionary of the English Language. ed 3. Boston: Houghton Mifflin; 1992. p. 1683.
2. van Dijk D, Keizer AMA, Diephuis JC, Durand C, Vos LJ, Hijman R. Neurocognitive dysfunction after coronary artery bypass surgery: a systematic review. J Thorac Cardiovasc Surg. 2000;120: 632-9.

3. Egger M, Smith GD. Meta-analysis: potentials and promise. $\mathrm{Br}$ Med J. 1997;315:1371-4.

4. Glass GV. Primary, secondary, and meta-analysis. Educational Researcher. 1976;5:3-8.

5. Lau J, Ioannidis JPA, Schmid CH. Quantitative synthesis in systematic reviews. Ann Intern Med. 1997;127:820-6. 